\title{
Particle-like Q-balls
}

\section{E.Ya. Nugaev ${ }^{a}$ and M.N. Smolyakov ${ }^{b}$}

${ }^{a}$ Institute for Nuclear Research of the Russian Academy of Sciences, 60th October Anniversary prospect 7a, 117312, Moscow, Russia

${ }^{b}$ Skobeltsyn Institute of Nuclear Physics, Lomonosov Moscow State University, 119991, Moscow, Russia

E-mail: emin@ms2.inr.ac.ru, smolyakov@theory.sinp.msu.ru

ABSTRACT: Usually the charge and the energy of stable Q-balls vary in a wide range or are even unbounded. In the present paper we study an interesting possibility that this range is parametrically small. In this case the spectra of stable Q-balls look similar to the one of free particles.

KEYwords: Solitons Monopoles and Instantons, Global Symmetries

ARXIV EPRINT: 1311.3418 
Among the variety of non-topological solitons (see [1, 2] for review) Q-balls [3, 4] and their properties were thoroughly examined, in particular, due to the interest encouraged by cosmology (see, for example, [5]). The main soliton characteristics, the energy $E$ and the charge $Q$, are functions of the parameter $\omega$ (the standard Q-ball solution in a scalar field theory with global U(1) invariance has the form $\left.\phi(t, \vec{x})=f(|\vec{x}|) e^{i \omega t}\right)$, which results in the possibility of different forms of $E(Q)$ dependence for different scalar field potentials.

Of course, the most interesting Q-ball solutions are stable solutions. In general, there are three types of the Q-ball stability:

1. The first type is the quantum mechanical stability, i.e., the stability with respect to decay into free particles. If $E(Q)<M Q$ for a Q-ball of charge $Q$, where $M$ is the mass of a free particle in the theory under consideration (without loss of generality, from here on we suppose that $\omega \geq 0$ and $Q \geq 0$ ), then such a Q-ball is quantum mechanically stable. ${ }^{1}$

2. The second type is the stability against fission. Q-balls are stable against decay into Q-balls with smaller charges if $d^{2} E / d Q^{2}<0$ (a simple justification of this fact in the general case can be found in [7]).

3. The third type is the classical stability, i.e., the stability with respect to small perturbations of the scalar field. The stability criterion proposed in $[2,8]$ implies that a Q-ball is classically stable if $\frac{d Q}{d \omega}<0$.

Below we will consider only those Q-ball solutions which satisfy all the three stability criteria, presented above. We will call them "absolutely stable" Q-balls. It should be noted that since the equality $\frac{d E}{d Q}=\omega$ always holds for Q-balls, the latter leads to

$$
\frac{d^{2} E}{d Q^{2}}=\frac{d \omega}{d Q}
$$

Thus, the criterion of stability against fission and the criterion of classical stability coincide, i.e., classically stable Q-balls are stable against fission.

Note that our definition of the absolute stability (at least in the absence of fermions) differs from the one of papers [9, 10], where the stability with respect to decay into free particles is supposed to be the strongest criterion, which Q-balls should satisfy, and such Q-balls are called absolutely stable in these papers. Our definition is different because, as we will see below, the stability with respect to decay into free particles does not imply the classical stability in the general case.

As it was noted above, the $E(Q)$ dependencies may have rather different forms in models with different potentials. As the first example one can recall the model presented in the well-known paper [8]. The $E(Q)$ dependence in this model consists of two branches, one of which (the lower one) is classically stable. Moreover, there exists $Q_{S}$ such that for $Q>Q_{S}$ the inequality $E(Q)<M Q$ holds for the lower branch (see figure 3(a) in [8]). Thus, Q-balls with $Q>Q_{S}$ from the lower branch of the $E(Q)$ dependence are absolutely

\footnotetext{
${ }^{1}$ In the case of special interactions with fermions this kinematic consideration should be revised, see [6].
} 
stable. An analogous form of the $E(Q)$ dependence is inherent to other models, see, for example, [7, 11-13].

Another type of Q-balls is the one with only one branch. As an example one may consider the model with $|\phi|^{4}$ potential studied in [14]. The $E(Q)$ dependence in this model consists of only one branch with $d^{2} E / d Q^{2}>0$, and all Q-balls in such a model are even classically unstable (this was also shown explicitly in [14]).

An interesting model with a logarithmic unbounded ${ }^{2}$ scalar field potential was proposed in [15] and thoroughly examined in [16]. The $E(Q)$ dependence in this model also consists of two branches, one of which is classically stable (again it is the lower branch). The charge of the Q-balls from the stable branch varies from 0 to $Q_{\max }<\infty$. An analogous $E(Q)$ behavior has the model with a simple polynomial potential discussed in [13].

In all the examples presented above the spectra of stable Q-balls (if they exist) either have no upper limit, or have an upper limit, but start from zero. In any case, the charge and the energy of such Q-balls vary in a wide range or are even unbounded. There arises a question: is it possible to make this range parametrically small?

To answer this question, we recall that in some models there is another form of the $E(Q)$ dependence. It consists of three branches, one of which, - the "lowest" branch, contains Q-balls which are classically stable. An important feature of this branch is that there exist both a lower bound on the charge $Q_{\min }$ and an upper bound $Q_{\max }$ such that Q-balls with $Q_{\min }<Q<Q_{\max }$ are classically stable. Such an $E(Q)$ dependence arises in the models with piecewise parabolic potentials examined in $[7,12]$ (these scalar field potentials were originally proposed in [3]), and in the model with a polynomial potential discussed in [13]. Below we will focus on examination of such an $E(Q)$ dependence with three branches.

In order to find out whether it is possible that the range of charges, where the absolutely stable Q-balls exist, can be made small, it is better to have an analytically solvable model. The models discussed in $[7,12]$ are analytically solvable (the model of [7] provides a very simple analytic Q-ball solution, which is very useful for examining perturbations above the Q-ball solution explicitly), but the scalar field potentials utilized in these models contain breaks, which is rather unphysical and demands an additional regularization of the potentials. Below we will propose a model with a continuous and differentiable potential, admitting the existence of a simple analytic Q-ball solution and providing the $E(Q)$ dependence with three branches, one of which corresponds to classically stable Q-balls. We will calculate $Q_{S}$ and $Q_{\max }$ in this model and answer the question posed above.

We consider the globally $\mathrm{U}(1)$ invariant scalar field theory with a piecewise potential of the form

$$
\begin{aligned}
V\left(\phi^{*} \phi\right)= & M^{2} \phi^{*} \phi \theta\left(v^{2}-\phi^{*} \phi\right) \\
& +\left(m^{2} \phi^{*} \phi+2 v\left(M^{2}-m^{2}\right) \sqrt{\phi^{*} \phi}-v^{2}\left(M^{2}-m^{2}\right)\right) \theta\left(\phi^{*} \phi-v^{2}\right),
\end{aligned}
$$

\footnotetext{
${ }^{2}$ Surely, one can add positive terms to the potential for very large values of the field modulus without altering the physics at the scale of stable Q-balls.
} 

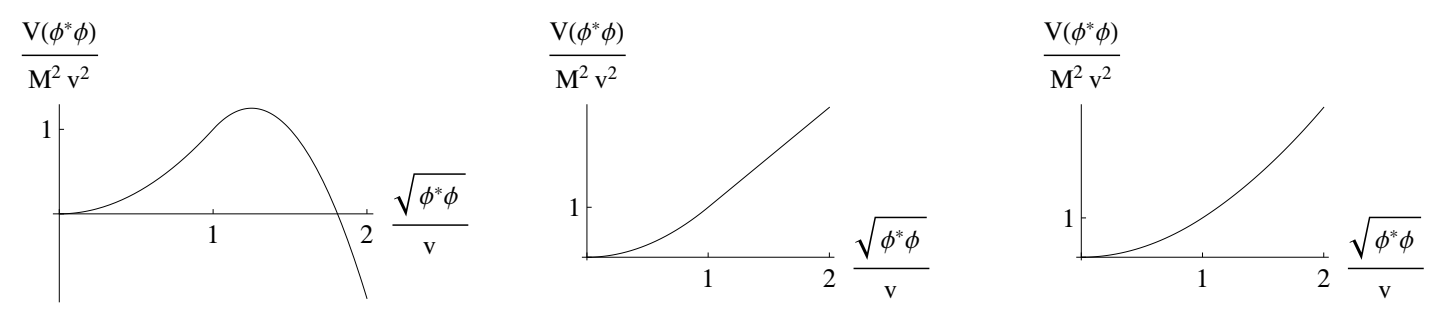

Figure 1. The forms of the scalar field potential described by eq. (2): $m^{2}<0,|m| / M=2$ (left plot); $m=0$ (middle plot); $m^{2}>0, m / M=0.9$ (right plot).

where $M^{2}>0, \theta$ is the Heaviside step function with the convention $\theta(0)=\frac{1}{2}$. The form of this scalar field potential for different values of the dimensionless parameter $\frac{m}{M}$ is presented in figure 1. We will be looking for a solution to the corresponding equation of motion of the standard form $\phi=f(r, \omega) e^{i \omega t}$, where $r=|\vec{x}|$. Without loss of generality, we suppose that $f(r, \omega)>0$. The monotonic solution for $f$ such that $\left.\frac{d f}{d r}\right|_{r=0}=0$ and $\left.f\right|_{r \rightarrow \infty}=0$ can be easily found and has the form

$$
\begin{array}{ll}
f(r, \omega)=v \frac{\left(M^{2}-m^{2}\right)}{\left(\omega^{2}-m^{2}\right)}-v \frac{\left(M^{2}-\omega^{2}\right)}{\left(\omega^{2}-m^{2}\right)} \frac{R}{r} \frac{\sin \left(\sqrt{\omega^{2}-m^{2}} r\right)}{\sin \left(\sqrt{\omega^{2}-m^{2}} R\right)}, & r<R, \\
f(r, \omega)=v \frac{R}{r} \frac{e^{-\sqrt{M^{2}-\omega^{2}} r}}{e^{-\sqrt{M^{2}-\omega^{2}} R}}, & r \geq R,
\end{array}
$$

where the matching radius $R$ is such that $f(R, \omega)=v$. For $r<R$ we have $f(r, \omega)>v$, whereas for $r>R$ we have $f(r, \omega)<v$. It is evident that if $m^{2}>0$, then $M>\omega>m$; if $m=0$, then $M>\omega>0$; otherwise $M>\omega \geq 0$.

The continuity of $f(r, \omega)$ and of its first derivative leads to the following equation for $R=R(\omega)$ :

$$
\left(\frac{M^{2}-m^{2}}{\omega^{2}-m^{2}}+\sqrt{M^{2}-\omega^{2}} R\right) \tan \left(\sqrt{\omega^{2}-m^{2}} R\right)=\frac{M^{2}-\omega^{2}}{\sqrt{\omega^{2}-m^{2}}} R
$$

This equation can be easily solved numerically for a given $\omega$. Note that equation (5) is valid only for Q-ball solutions without nodes. For such a solution and for a given $\omega$ one should take the first (smallest) root of (5) satisfying the condition $R(\omega)>\frac{\pi}{\sqrt{\omega^{2}-m^{2}}}$.

The Q-ball charge and energy can also be easily calculated and have the form

$$
\begin{aligned}
& Q=2 \omega \int_{0}^{\infty} f^{2} d^{3} x \\
&=4 \pi \omega v^{2}\left[\frac{R^{3}}{\left(\omega^{2}-m^{2}\right)^{2}}\left(\frac{2}{3}\left(M^{2}-m^{2}\right)^{2}+\left(M^{2}-\omega^{2}\right)^{2}+\left(\omega^{2}-m^{2}\right)\left(M^{2}-\omega^{2}\right)\right)\right. \\
&\left.\left.\quad+\frac{R^{2} \sqrt{M^{2}-\omega^{2}}}{\left(\omega^{2}-m^{2}\right)^{2}}\left(5 M^{2}-6 m^{2}+\omega^{2}\right)\right)+5 \frac{R\left(M^{2}-m^{2}\right)}{\left(\omega^{2}-m^{2}\right)^{2}}+\frac{R^{2}}{\sqrt{M^{2}-\omega^{2}}}\right], \\
& E=\omega Q+4 \pi v^{2} \frac{M^{2}-m^{2}}{\omega^{2}-m^{2}}\left[\frac{R^{3}}{3}\left(M^{2}-\omega^{2}\right)+R^{2} \sqrt{M^{2}-\omega^{2}}+R\right],
\end{aligned}
$$

where we have used eq. (5) in the derivation. 

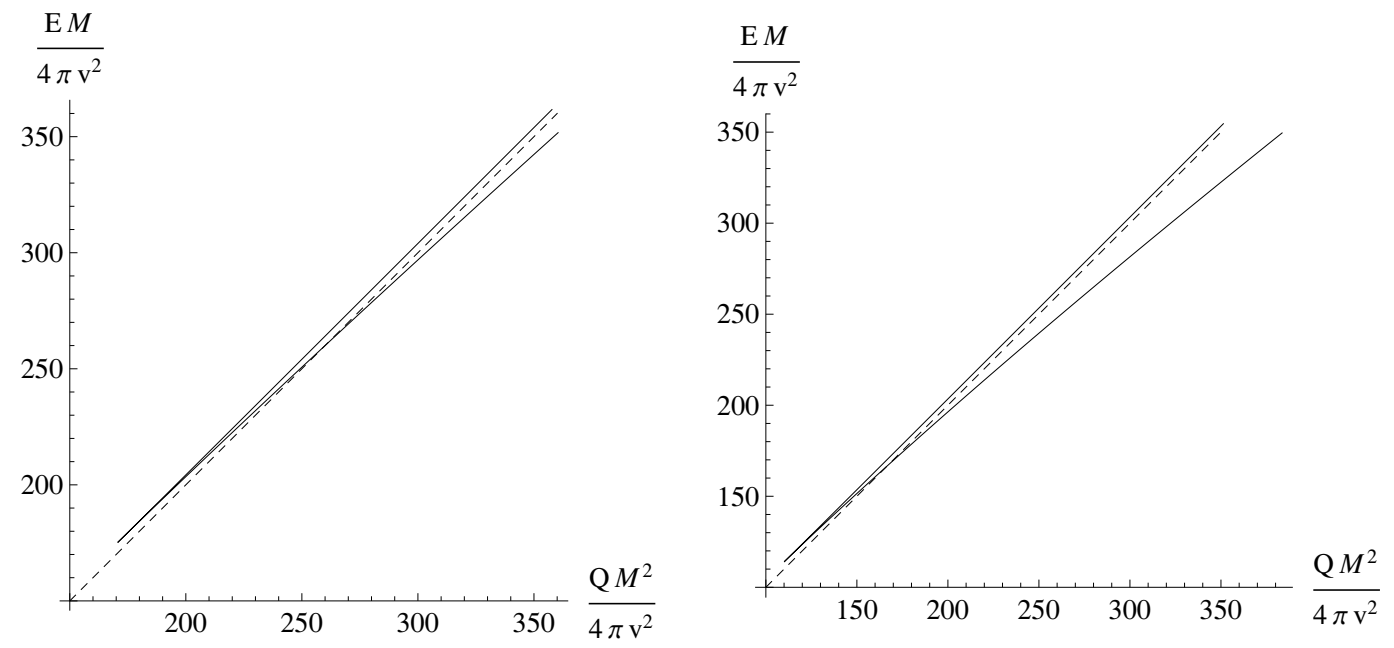

Figure 2. $\mathrm{E}(\mathrm{Q})$ for $m^{2}>0, \frac{m}{M}=0.5$ (left plot) and for $m=0$ (right plot). The dashed line corresponds to free scalar particles of mass $M$.

Now let us examine the $E(Q)$ dependence for different values of the model parameters, i.e., for $m^{2}>0, m=0$ and $m^{2}<0$. It is not difficult to show that the charge (6) and the energy (7) can be represented as

$$
Q=\frac{4 \pi v^{2}}{M^{2}} \tilde{Q}, \quad E=\frac{4 \pi v^{2}}{M} \tilde{E}
$$

where $\tilde{Q}$ and $\tilde{E}$ depend only on $\frac{\omega}{M}$ and $\frac{m}{M}$ and do not depend on $v$. So, below we will not specify the values of $v$ and $M$ while examining the main properties of Q-balls in our model: the $E(Q)$ dependencies can be examined by considering the dimensionless quantities $\tilde{Q}$ and $\tilde{E}$ for different choices of $\frac{m}{M}$. Such a simplification is possible only because of the simple form of the scalar field potential, which appears to be very useful for calculations.

The corresponding plots are presented in figures 2 and 3 . We see that the $E(Q)$ diagrams for the cases $m^{2}>0$ and $m=0$ resemble those in the models discussed in $[8,11-$ 13]. All four cases, presented in figures 2 and 3, also exist in the model discussed in [7]. It should be noted that, though $\omega$ is bounded from above, $\omega<M$, in the limit $\omega \rightarrow M$ the charge and the energy tend to infinity in all four cases, presented in figures 2 and 3 . This happens because the factor $\sqrt{M^{2}-\omega^{2}}$ in the exponent of (4) tends to zero for $\omega \rightarrow M$, whereas $\left.R(\omega)\right|_{\omega \rightarrow M} \rightarrow \frac{\pi}{\sqrt{M^{2}-m^{2}}}$; so the scalar field falls off not exponentially, but as $\frac{1}{r}$ in this limit. The latter leads to infinite charge and energy of the Q-ball for $\omega \rightarrow M$. Due to the large size of the Q-ball core, such Q-balls were called "Q-clouds" in [11].

As it was noted above, we will be interested in the last case $m^{2}<0$. As it can be seen from figure 3, there are two phases: the first phase contains three branches on the $E(Q)$ diagram, whereas the other phase contains only one branch (the latter case is similar to the one of the model with $|\phi|^{4}$ potential studied in [14]). The transition between the phases occurs at $\frac{|m|}{M} \approx 1.775$. One also sees from the left plot in figure 3 that the most part of the lowest stable branch lies under the $E=M Q$ line corresponding to free particles, which 

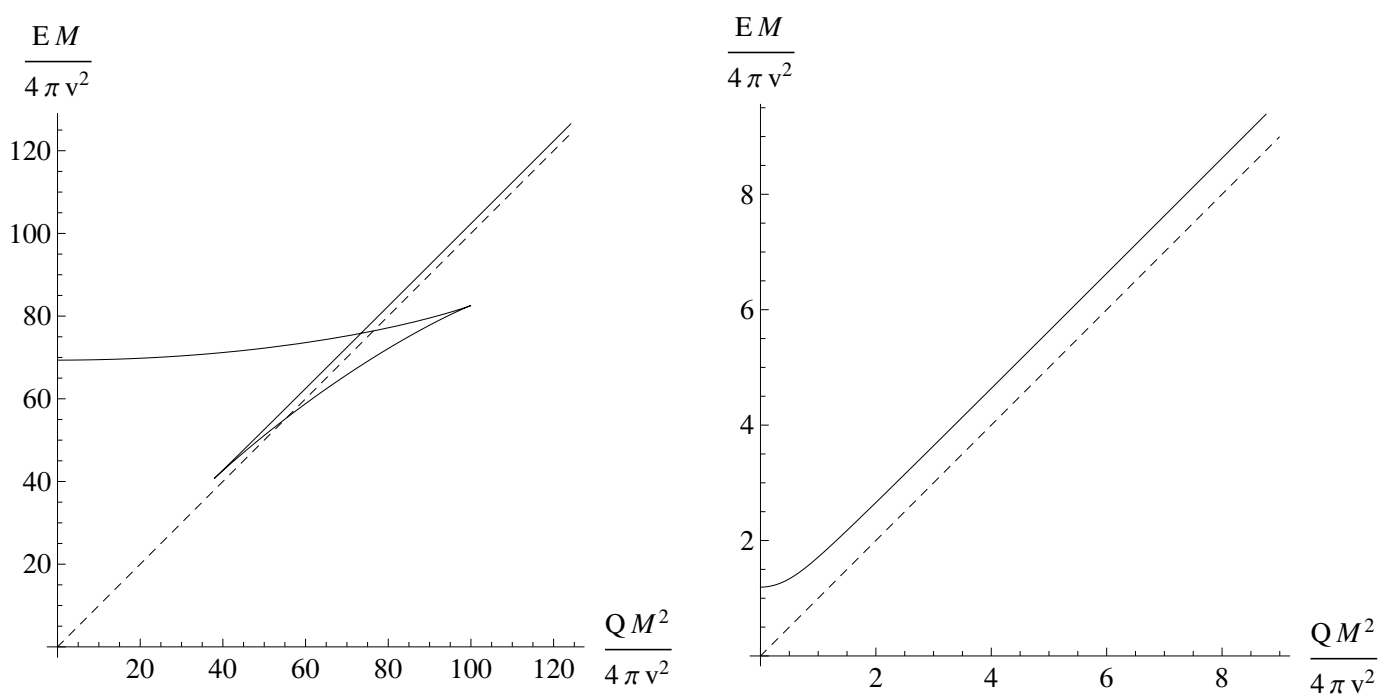

Figure 3. $\mathrm{E}(\mathrm{Q})$ for $m^{2}<0 . \quad \frac{|m|}{M}=1$ (left plot) and $\frac{|m|}{M}=5$ (right plot). The dashed line corresponds to free scalar particles of mass $M$.

means that the range of charges of absolutely stable Q-balls is rather large. Note that the part of the upper classically unstable branch (which starts from $Q=0$ ) on the left plot in figure 3 also lies under the $E=M Q$ line corresponding to free particles, which means that the stability with respect to decay into free particles indeed does not imply the classical stability in the general case.

We would like to note that the existence of a locally maximal charge in the phase with three branches (see figure 3) seems to be a consequence of the turnover of the scalar field potential. We think that this is a rather general property, which is inherent to other models of Q-balls. Although we can not prove it in a rigorous way, we do not know exceptions from this rule. Meanwhile, the opposite is not correct - the existence of the turnover of the scalar field potential does not guarantee the existence of a locally maximal charge, which is confirmed by the existence of the phase without maximal charge for $\frac{|m|}{M}>1.775$ in our case and by the examples of other models (see, for example, [14]). We also stress that the maximal value of $f(r, \omega)$ (which is simply $f(0, \omega))$ of the Q-ball with locally maximal charge is not connected with the point of the maximum of the scalar field potential for $m^{2}<0$. Indeed, the scalar field potential is maximal at $f_{\mathrm{Vmax}}=v\left(1+\frac{M^{2}}{|m|^{2}}\right)$; whereas the value of $f(0, \omega)$ decreases monotonically (this can be checked numerically) from $f(0,0)$ to $f(0, M)=2 v$. So, if $\frac{|m|}{M} \geq 1$ (see, for example, left plot in figure 3 ), then $f(0, \omega)>2 v \geq f_{\mathrm{Vmax}}$ : the maximum of the absolute value of the Q-ball scalar field is larger than the point of the maximum of the scalar field potential for any $0 \leq \omega<M$, i.e., for any Q-ball. An interesting observation in the opposite case $\frac{|m|}{M}<1$ is that Q-balls with $\omega \rightarrow M$ lie on the unstable branch, whereas $f(0, M)=2 v<f_{\mathrm{Vmax}}$ in this case. These examples demonstrate that there is no (at least obvious) connection between the maximal absolute value of the Q-ball scalar field, the point of the maximum of the scalar field potential and the Q-ball stability. 
(a)

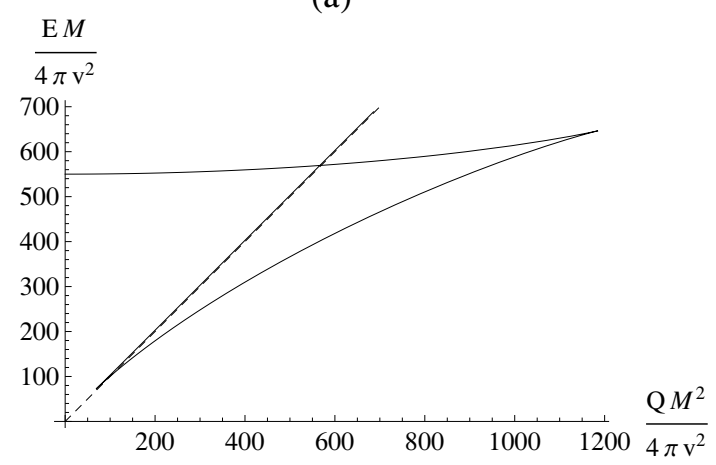

(c)

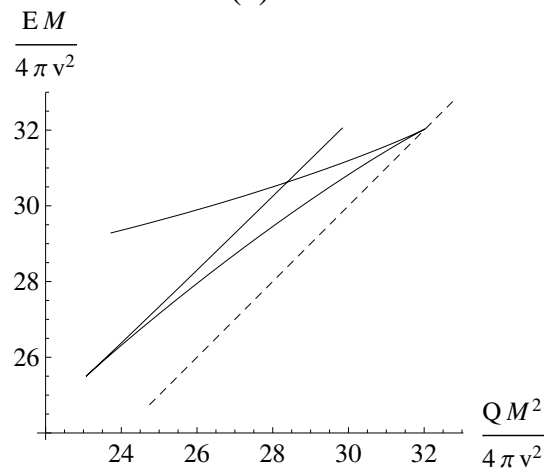

(b)

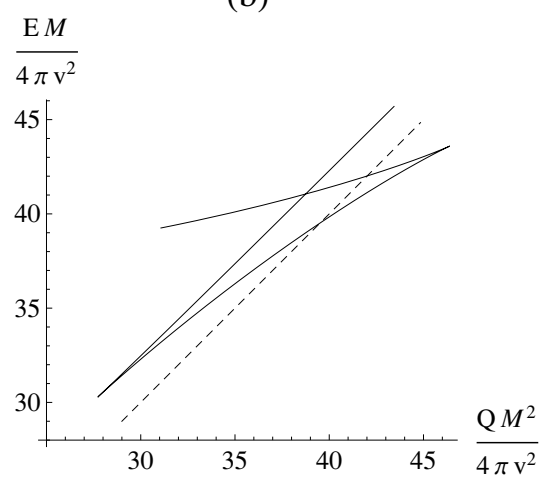

(d)

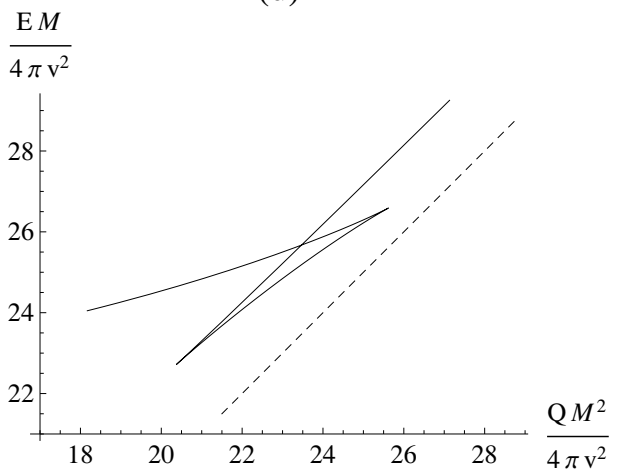

Figure 4. $\mathrm{E}(\mathrm{Q})$ for $m^{2}<0 . \frac{|m|}{M}=0.6$ (a); $\frac{|m|}{M}=1.2(\mathrm{~b}) ; \frac{|m|}{M}=1.31886$ (c); $\frac{|m|}{M}=1.4$ (d). The dashed line stands for free particles of mass $M$.

Now let us check what happens when we change the parameter $\tilde{m}=\frac{|m|}{M}$. The result is presented in figure 4 . We see that the larger $\tilde{m}$ is, the smaller the "triangle" in the corresponding $E(Q)$ diagram is. Moreover, the larger $\tilde{m}$ is, the smaller part of this "triangle" turns out to lie under the $E=M Q$ line corresponding to free particles. For $\tilde{m}=\tilde{m}_{x} \approx 1.31886$ the "triangle" touches the free particles line by the upper cusp, whereas for $\tilde{m}>\tilde{m}_{x}$ all the classically stable Q-balls are quantum mechanically unstable. For $\tilde{m} \gtrsim 1.775$ the "triangle" disappears and there is no classically stable branch in the $E(Q)$ dependence at all.

The observations presented above indicate that there exist such values of the parameters that absolutely stable Q-balls can exist only in a very narrow range of charges (and, consequently, energies). As an example, let us take $\tilde{m}=1.315$. The upper right part of the "triangle", including the cusp, is presented in figure 5. The lower branch in this figure is the stable one. The values of $\tilde{Q}=\frac{Q M^{2}}{4 \pi v^{2}}$ and $\tilde{E}=\frac{E M}{4 \pi v^{2}}$, corresponding to the dots on the plot, are the following: $\tilde{Q}_{S} \approx 32.268, \tilde{E}_{S} \approx 32.268, \tilde{Q}_{\max } \approx 32.394, \tilde{E}_{\max } \approx 32.334$. The ranges of the charges and the energies, for which the absolutely stable Q-balls can exist, are

$$
\Delta \tilde{Q}=\tilde{Q}_{\max }-\tilde{Q}_{S} \approx 0.126, \quad \Delta \tilde{E}=\tilde{E}_{\max }-\tilde{E}_{S} \approx 0.066
$$

These ranges are much smaller than the absolute values of the charges and the energies respectively. 


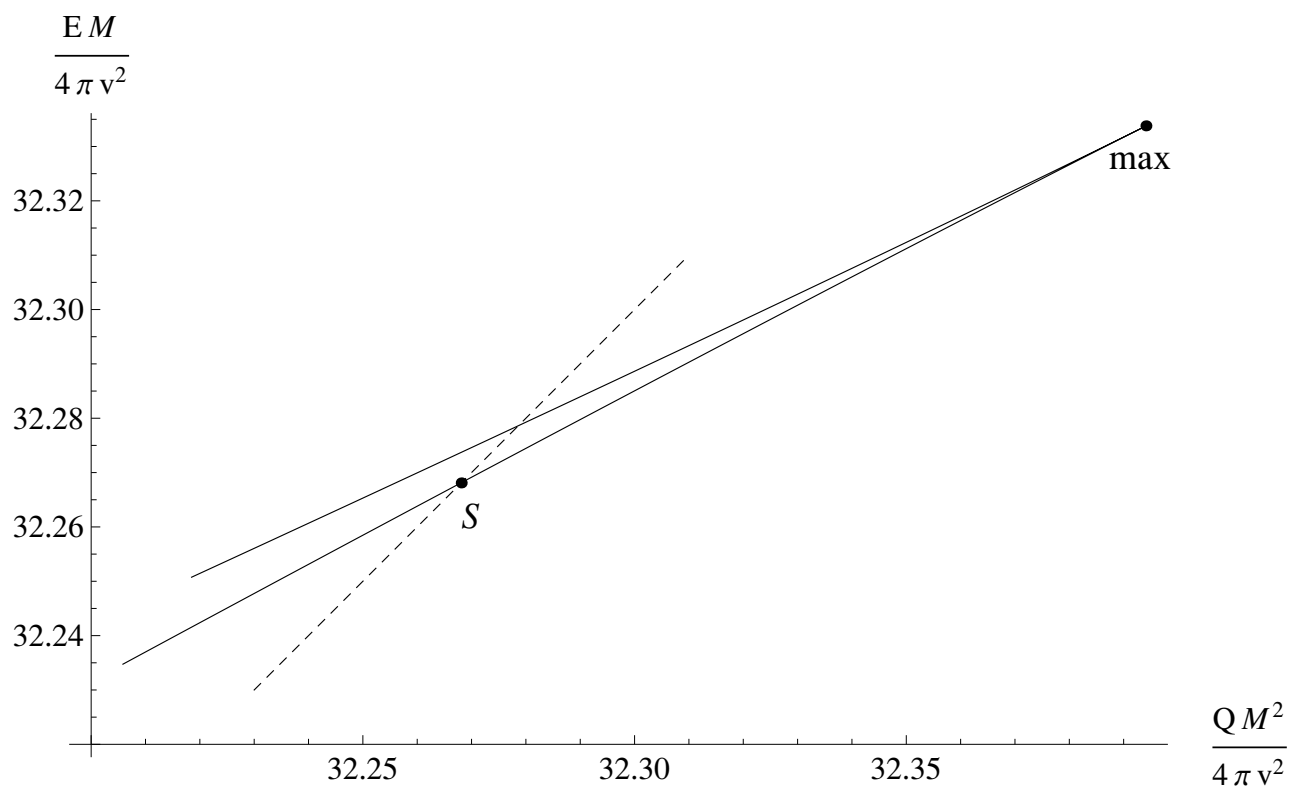

Figure 5. $\mathrm{E}(\mathrm{Q})$ for $m^{2}<0 . \frac{|m|}{M}=1.315$. The dashed line stands for free particles of mass $M$.

The closer (from below) $\tilde{m}$ to $\tilde{m}_{x} \approx 1.31886$ is, the smaller $\Delta \tilde{Q}$ and $\Delta \tilde{E}$ are. For $\Delta \tilde{Q} \ll \tilde{Q}_{\max }$ the $E(Q)$ dependence of the absolutely stable Q-balls is similar to the one in the limiting case $\tilde{m} \rightarrow \tilde{m}_{x}$ :

$$
E=M Q_{x},
$$

where $\tilde{Q}_{x}=\left.\tilde{Q}_{\max }\right|_{\tilde{m}=\tilde{m}_{x}} \approx 32.034$. But it looks exactly like the $E(Q)$ dependence of free particles at rest! The only difference is that the charge of free particles $Q_{p}=1$, whereas for Q-balls we have in the limiting case $Q_{x} \approx 32 \frac{4 \pi v^{2}}{M^{2}}$. Of course, analogous anti-Q-balls (i.e., Q-balls with $\omega<0$ and $Q<0$ ) also exist and possess the same properties.

We think that the existence of such particle-like Q-balls should be inherent not only to the model presented above, but to other models providing an $E(Q)$ dependence with three branches (namely, to models with scalar field potential admitting the existence of a true vacuum at $\phi^{*} \phi>0$ or at least having a negative slope after some nonzero value of the scalar field modulus), like those in $[7,12,13]$. In such models there is a possibility to tune the charge of a stable Q-ball to a nearly determined value, i.e., it is possible to have absolutely stable Q-balls with the ranges of charges and energies much smaller than the absolute values of charges and energies themselves. The spectra of such Q-balls are very similar to the spectrum of free particles of the theory and Q-balls behave like clusters of free particles, which looks very intriguing.

\section{Acknowledgments}

The authors are grateful to D. Levkov, M. Libanov and I. Volobuev for discussions and to the unknown referee for useful comments. The work was supported by RFBR grant 14-02-31384. The work of E.Y.N. was supported in part by grant NS-2835.2014.2 of the President of Russian Federation and by RFBR grant 13-02-01127a. The work of M.N.S. 
was supported in part by grant NS-3042.2014.2 of the President of Russian Federation and by RFBR grant 12-02-93108-CNRSL-a.

Open Access. This article is distributed under the terms of the Creative Commons Attribution License (CC-BY 4.0), which permits any use, distribution and reproduction in any medium, provided the original author(s) and source are credited.

\section{References}

[1] V.G. Makhankov, Dynamics of classical solitons in nonintegrable systems, Phys. Rept. 35 (1978) 1 [inSPIRE].

[2] T.D. Lee and Y. Pang, Nontopological solitons, Phys. Rept. 221 (1992) 251 [InSPIRE].

[3] G. Rosen, Particlelike solutions to nonlinear complex scalar field theories with positive-definite energy densities, J. Math. Phys. 9 (1968) 996.

[4] S.R. Coleman, Q-balls, Nucl. Phys. B 262 (1985) 263 [Erratum ibid. B 269 (1986) 744] [INSPIRE].

[5] D.S. Gorbunov and V.A. Rubakov, Introduction to the theory of the early universe: Hot big bang theory, World Scientific, U.S.A. (2011).

[6] A.G. Cohen, S.R. Coleman, H. Georgi and A. Manohar, The evaporation of $Q$ balls, Nucl. Phys. B 272 (1986) 301 [InSPIRE].

[7] I.E. Gulamov, E.Y. Nugaev and M.N. Smolyakov, Analytic Q-ball solutions and their stability in a piecewise parabolic potential, Phys. Rev. D 87 (2013) 085043 [arXiv: 1303.1173] [INSPIRE].

[8] R. Friedberg, T.D. Lee and A. Sirlin, A class of scalar-field soliton solutions in three space dimensions, Phys. Rev. D 13 (1976) 2739 [InSPIRE].

[9] M.I. Tsumagari, E.J. Copeland and P.M. Saffin, Some stationary properties of a Q-ball in arbitrary space dimensions, Phys. Rev. D 78 (2008) 065021 [arXiv:0805.3233] [INSPIRE].

[10] M.I. Tsumagari, The physics of Q-balls, arXiv:0910.3845 [INSPIRE].

[11] M.G. Alford, Q-clouds, Nucl. Phys. B 298 (1988) 323 [inSPIRE].

[12] S. Theodorakis, Analytic Q-ball solutions in a parabolic-type potential, Phys. Rev. D 61 (2000) 047701 [INSPIRE].

[13] T. Tamaki and N. Sakai, Unified pictures of Q-balls and Q-tubes, Phys. Rev. D 86 (2012) 105011 [arXiv:1208.4440] [INSPIRE].

[14] D.L.T. Anderson and G.H. Derrick, Stability of time-dependent particlelike solutions in nonlinear field theories. 1, J. Math. Phys. 11 (1970) 1336 [INSPIRE].

[15] G. Rosen, Dilatation covariance and exact solutions in local relativistic field theories, Phys. Rev. 183 (1969) 1186 [INSPIRE].

[16] G.C. Marques and I. Ventura, Resonances within nonperturbative methods in field theories, Phys. Rev. D 14 (1976) 1056 [INSPIRE]. 